\title{
Recent development of GC capillary columns in Japan
}

\author{
C. Watanabe ${ }^{1}$, Y. Kawahara ${ }^{1}$ and K. Jinno ${ }^{2}$ \\ ${ }^{1}$ R\&D, Frontier Laboratories Ltd., 61-2 Otsubo, Otsuki, Koriyama 963-0201, Japan \\ ${ }^{2}$ School of Material Science, Toyohashi University of Technology, Toyohashi 941-8580, Japan
}

\begin{abstract}
Because of the epoch-making inner surface inertness and excellent properties such as bending strength or flexibility, the FS columns have made a great contribution to progress of GC analysis. Maintaining such satisfactory basic properties, withstanding at elevated high temperatures is required for GC capillary columns, having an inner structure resistant to the buildups, and offering a high sample loading capacity. The highly inert metallic capillary columns developed in Japan fulfills all of these requirements and it seems to be very promising as the main GC separation columns in the future.
\end{abstract}

apillary columns are considered to be the heart of gas chromatographs (GC) as software in computer sciences. Since M.J.E. Golay invented capillary columns, about 40 years have elapsed. Especially in the decade from 1978, the performance of columns has remarkably improved in terms of column materials as well as the quality of stationary phases. Various injection methods such as split/splitless, on-column and programmable temperature vaporizer as well as detectors have also been developed and improved to match the improved high column performances. Assisted also by the astonishing progress in microelectronics, the overall capabilities of GC have been dramatically enhanced to date.

Materials used for separation columns have historically shifted from metals (mainly stainless steel) to glass and then to fused silica (FS). Metal columns are recently spotlighted again in Japan. This article describes the present situation on GC capillary columns in Japan and introduces the deactivated metallic capillary columns often called as columns for the next generation. We would like to introduce their performances compared to those of fused silica capillary columns.

\section{Capillary columns and their situation in Japan}

\section{Fused silica columns and their situation in Japan}

Majority of the FS capillary columns presently used in all over the world are made out of very high purity fused silica materials employed for the optical fibers. It is, therefore, considered to possess an ultimate quality. Dandeneau and others invented easy-handling columns that are suitable for practical uses. They have applied a coating of polymers such as polyimide, a highly thermally stable engineering polymer, on the external surface of a FS tube with about $0.1 \mathrm{~mm}$ wall thickness and drastically improved the bending resistance and flexibility [1]. This coating prevented the detrimental loss of mechanical strengths due to mechanical scratches or penetration of salt in sweat into the silica network structure. After the appearance of these columns, GC analysis utilizing FS columns coated with only a few kind of stationary phases became available for relatively inexperienced analysts, while a certain level of skills and experiences were required to use packed columns.

During the 15 years after the FS columns came to practical uses, demands appeared to further improvement of excellent FS columns already used.

The first demand was to improve the outer wall of FS columns in thermal stability. Basic research has been advanced toward developing stationary phases that are stable at elevated temperature and capillary columns made from glass usable at temperatures higher than $400{ }^{\circ} \mathrm{C}$ have appeared. As the polyimide protective coating for the column can stand normally up to around $330{ }^{\circ} \mathrm{C}$, attempts were made using modified polyimides with a greater heat stability or with aluminum clad columns. However, the column breakage problem due to the deterioration of the coating has not been solved to date, because the column faces high temperature in air circumstance causing thermal degradation and a rapid heating and cooling causing thermal expansion and shrinkage of the coating material that can not be escaped from the resulting stress cracking.

The second demand is to eliminate the weakness of FS columns in becoming dirty due to the build-up of high boiling point materials in a sample being analyzed. The FS columns are much worse in this respect than packed columns. No reasonable solution has been found yet, however.

The third point is about its small sample capacity, which is about hundred times smaller than that of packed columns. So-called wide-bore capillary columns with $0.53 \mathrm{~mm}$ i.d. are sometimes used in place of packed columns. Because they can accept a flow rate greater than $10 \mathrm{~mL} / \mathrm{min}$, wide-bore columns are easily handled and offering their high separation capability with long column length in addition to its ultimate inertness. They will be much more useful if an improvement is made in their sample capacity. 
Development and manufacturing of FS capillary columns are not carried out in Japan. As the patent situation prohibits domestic manufacturing, Japanese companies import most of them by any companies from the USA. They are widely used in various analytical applications in petroleum chemistry, natural product chemistry, environmental science, research and development and even in quality control in manufacturing plants. However, packed columns still have about $30 \%$ of the share in quality control applications at factories. Several reasons can be considered for this fact. When a capillary column is used to determine the purity of a product, one can not escape from troublesome operations associated with different injection methods in order to use an appropriate one. This might be one of the reasons.

One of the demands mentioned above, how to increase the sample capacity, has been solved in Japan by increasing the i.d. of the column to $1.2 \mathrm{~mm}$, although the mechanical strength problem is still remained as the unsolved because of the glass as the material. This type of columns is becoming popular because the flow rates similar to those for the packed columns and injecting a large amount of sample would not produce any problem.

\section{Development of metallic capillary columns and their characteristics}

The requirement of columns to overcome above-mentioned shortcomings of FS capillary columns are summarized as follows; (1) columns withstanding elevated temperatures, (2) columns enduring build-ups of high-boilers as much as packed columns can, and (3) columns with a large sample capacity. Recently, capillary columns satisfying all these requirements the technology to fulfill the above requirements have been developed in Japan.

When a capillary column is made from metal, it has excellent resistance to mechanical bending, impact and thermally stable at any temperature in GC operation. Based on the results of fundamental study by Takayama and others in Japan [2], it has been found that inert capillary columns made from metal are usable at temperatures above $400{ }^{\circ} \mathrm{C}$ and put in practical uses first time in the world in 1986 [3]. Further developments to improve their performance for commercialization in inertness and separation power of metallic capillary columns were made and now widely employed throughout Japan. The profile is briefly introduced here.

\section{Columns withstanding elevated temperatures with high inertness and separation power}

On the inner surface of a metallic capillary tube, special slanted 5-7 multi-layers are applied of which model is shown in figure 1 . The neighboring layers are chemically bonded by proprietary technology. The chemical composition of each layer gradually shifts to the next layer in a gradient manner. Applying this modification of its active surface, the metallic surface is hidden under the multi layers. Figure 2 shows the analytical result of the upper layer by the Auger spectroscopy indicating that its surface is a very thin film of silicon oxide and the inside is silicone. In other words, this tubing has an ideal construction with the inner surface being a very thin layer of fused silica and the exterior being stainless steel. After this modification, deactivation of surface and coating and immobilization of the stationary phase are applied for producing the capillary columns. Metalic capillary columns could be made by other methods such as coating the inner wall of a metallic capillary tube with a thick layer of fused silica. When this column is bent, the inner FS wall is vulnerable to break and
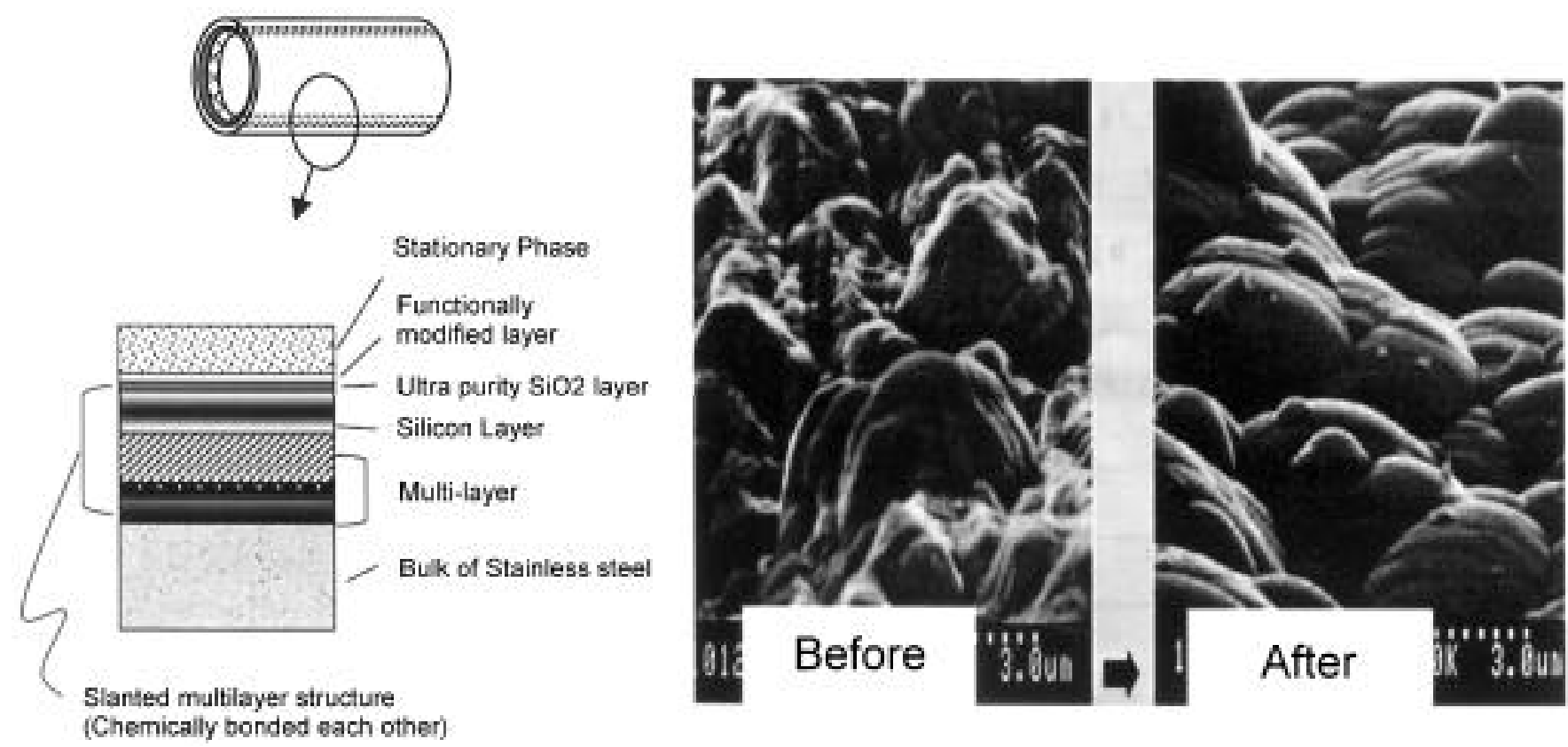

Figure 1. Model of stainless steel Ultra ALLOY capillary column by slanted chemical bonding technology and its SEM images of its surface before and after modification. 
metallic surface is exposed. The column will then be likely to end up with showing a very high adsorption to any polar components as shown in figure 3. In this figure, Ultra ALLOY slanted multi-layer column shows no deterioration after venting the inlet of the column. Such defects can not be detected from the appearance and this type of columns will be unsuitable for the GC analysis.

As the metallic Ultra ALLOY capillary columns having the inert characteristics as shown in figure 1 can be preconditioned by baking at $400{ }^{\circ} \mathrm{C}$ for over night under He carrier gas flow, its bleeding is minimized even at a high temperature.

\section{Columns enduring build-ups}

Because of its very smooth inner wall surface, FS columns are subject to be affected by the build-ups much more than the packed columns. Column packing materials in the

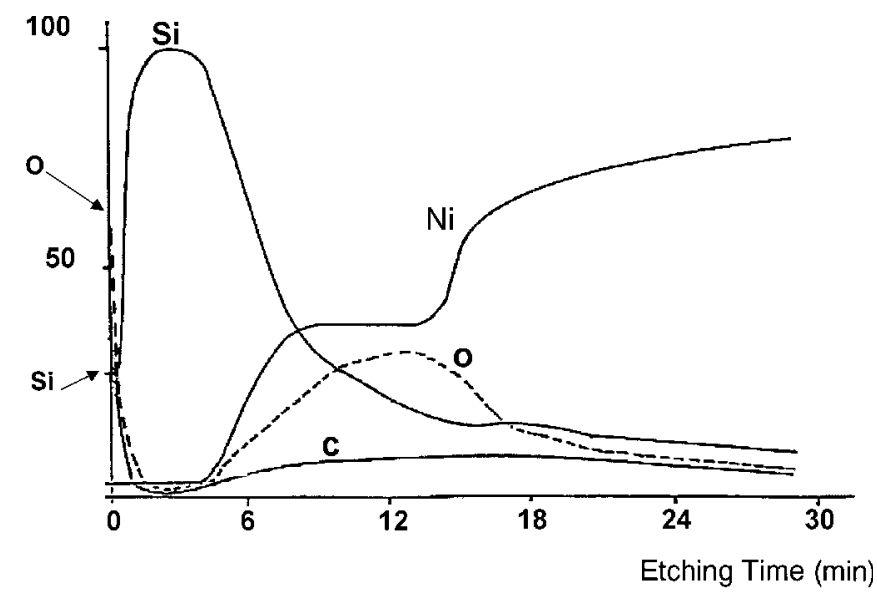

Figure 2. Elemental analysis of modified SS surface by Auger analysis.

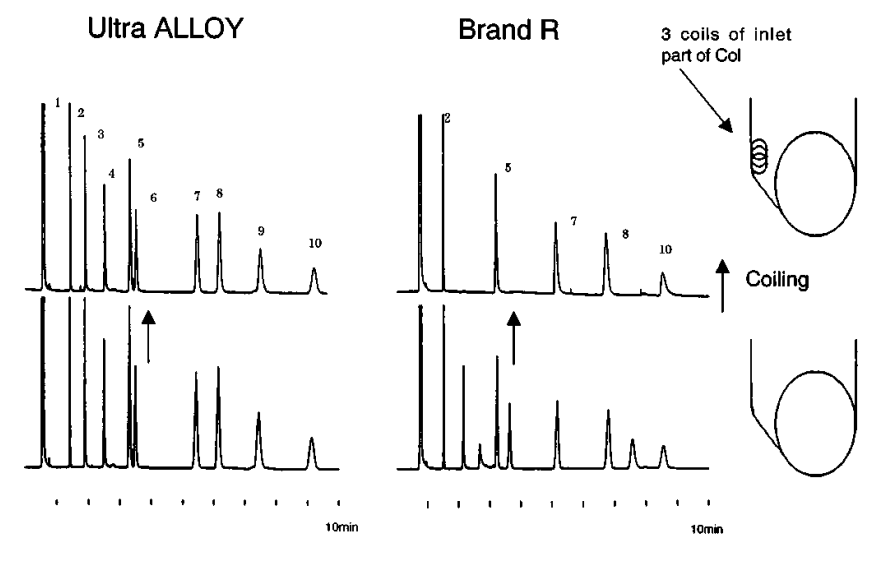

Figure 3. Changing the inertness by coiling a part of each column producing two different surface treated SS column. Column: $5 \%$ diphenylsilicone, $15 \mathrm{~m} \times 0.5 \mathrm{~mm}$ i.d. $(0.5 \mu \mathrm{m}$ film). Temperature: $120{ }^{\circ} \mathrm{C}$. Samples: 1=solvent; $2=$ =undecane; 3=4chlorophenol; 4=1-decylamine; $5=$ tridecane; $6=$ methylcaprate; 7=tetradecane; 8=acenaphthylene; 9=1-dodecanol; 10=pentade- packed column can withstand the build-ups since they have very high surface areas. For instance, the inner surface area of an FS columns of $30 \mathrm{~m}$ long $\times 0.25 \mathrm{~mm}$ i.d., is less than about $1 / 250$ of that of a packed column, $2 \mathrm{~m}$ long $\times 2 \mathrm{~mm}$ i.d., packed with a 80/100 mesh packing material. As the metallic capillary column has very rough inner surface as seen in figure 1, its surface area is about 20 times larger than that of FS column. This difference in surface area contributes to the metallic capillary column showing a better build-up resistance than FS columns. Figure 4 shows the comparison of their deterioration after repetitive injection of a standard sample added with $5 \%$ of sesame oil. The FS column lost its separation ability after 20 injections, while the metallic capillary column withstood four times longer. In another example of capillary GC/MS analysis of metabolites in several thousands blood and urine samples in order to detect congenital diseases, the metallic column was used for about 2000 sample injections, while the FS column was failed after 400 sample injections.

\section{Ultra ALLOY}
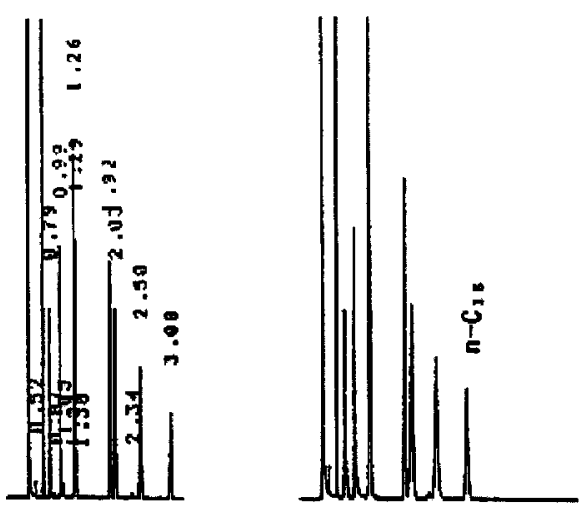

First inj. After 82nd inj.

\section{FS Brand J}

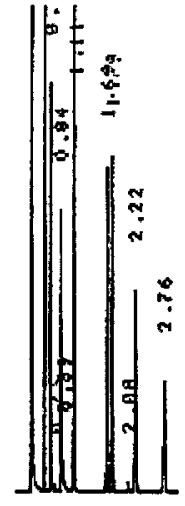

First Inj

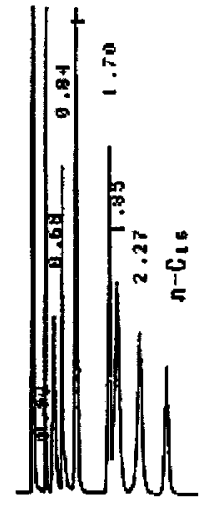

21 Inj.
Figure 4. Durability contamination test with metal and fused silica capillary column. Column: dimethylpolysiloxane, $10 \mathrm{~m} \times \mathbf{0 . 2 5}$ $\mathrm{mm}$ i.d. $\left(0.25 \mu \mathrm{m}\right.$ film). Temperature: $120{ }^{\circ} \mathrm{C}$. Sample: $1 \mu \mathrm{L}$ split injection, same samples as in figure 3 (added $5 \%$ sesame oil). 

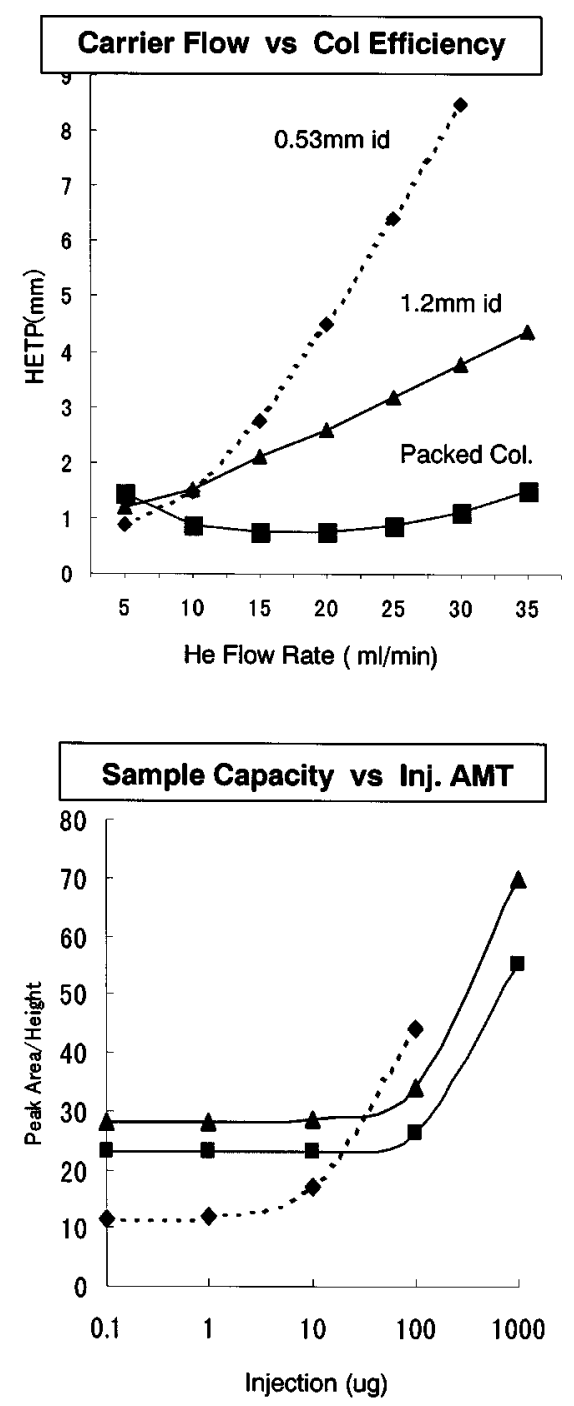

Figure 5. Column efficiency and sample loading capacity of various columns. Columns: dimethylpolysiloxane, $30 \mathrm{~m} \times$ $0.53 \mathrm{~mm}$ i.d. (1 $\mu \mathrm{m}$ film); $20 \mathrm{~m} \times 1.2 \mathrm{~mm}$ i.d. $(1 \mu \mathrm{m}$ film); packed, $2 \mathrm{~m} \times 2.2 \mathrm{~mm}$ i.d. (5\% OV-1). Sample: pentadecane.

\section{Columns with a high sample capacity}

Sample capacity of the column for the compounds is proportional to the square of column i.d., amount of the stationary liquid phase, and its length. In order to increase the sample capacity, the use of larger inner diameter columns is easy task for this requirement. However FS capillary column

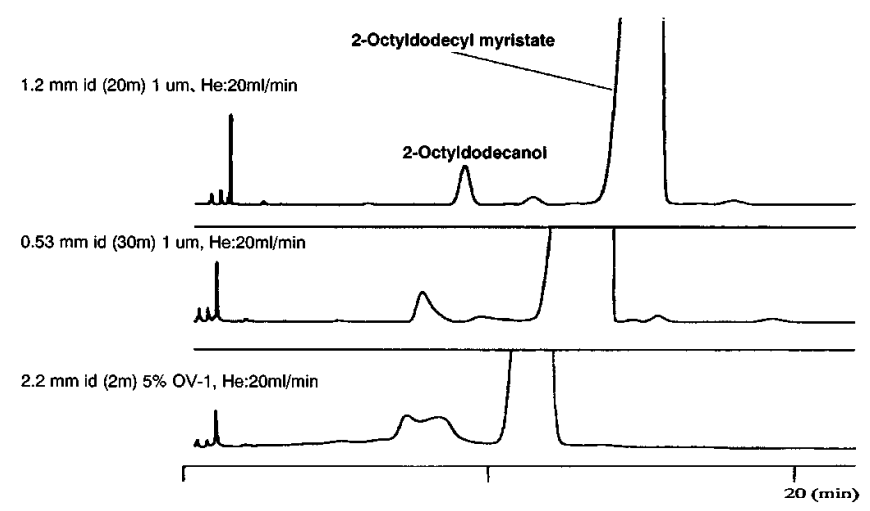

Figure 6. Analysis of unreacted alcohol in 2-octyldodecyl myristate. Columns: $20 \mathrm{~m} \times 1.2 \mathrm{~mm}$ i.d. $(1 \mu \mathrm{m}$ film); $30 \mathrm{~m} \times 0.53 \mathrm{~mm}$ i.d. $(1 \mu \mathrm{m}$ film); packed, $2 \mathrm{~m} \times 2.2 \mathrm{~mm}$ i.d. $(5 \%$ OV-1). Carrier gas: helium. Temperature: $270{ }^{\circ} \mathrm{C}$. Sample: cosmetic ingredient, $0.2 \mu \mathrm{L}$.

cannot be produced as a tubing larger than $0.53 \mathrm{~mm}$ i.d. due to the limitation of mechanical strength but it is not applied for metallic tubing. Figure 5 shows the relationship of column efficiency and sample loading capacity using different size columns including a newly developed one with $1.2 \mathrm{~mm}$ i.d. The properties of this column indicates that the column efficiency is better than that with a half i.d. capillary when the flow rate of carrier gas is greater than $15 \mathrm{~mL} / \mathrm{min}$. It also appears that the metallic capillary column with $1.2 \mathrm{~mm}$ i.d. has about five times larger sample loading capacity than that of a column with $0.53 \mathrm{~mm}$ i.d. and the performance in approaching to that with a packed column.

In the analysis of a trace amount of unreacted alcohol in a long-chain fatty acid ester used in cosmetic application as seen in figure 6, a FS column with $0.53 \mathrm{~mm}$ i.d. showed a broad peak even if the same alcohol in an identical concentration in hexane as solvent was not eluted with any tailing at all. This is considered due to the small sample loading capacity. The main ester component produced a broad band at the inlet of the column and influenced the peak shape of the alcohol.

\section{References}

1. Dandeneau, R. D.; Zerenner, E. H. HRC\&CC 1979, 2, 563.

2. Takayama, Y.; Yakeichi, T.; Kawai, S. HRC\&CC 1988, 11, 732.

3. Brochure of Metal capillary column of Nippon Chromato Company 1986, Tokyo, Japan. 